UCRL-JC-129503

PREPRINT

\title{
Status of Experiments Leading to a Small Recirculator
}

\section{T. Sangster, J. J. Barnard, T. V. Cianciolo, G. D. Craig, A. Friedman,} D. P. Grote, E. Halaxa, R. L. Hanks, G. Kamin, H. C. Kirbie, B. G. Logan, S. M. Lund, G. Mant, A. W. Molvik, W. M. Sharp, S. Eylon, D. Berners, T. J. Fessenden, D. L. Judd, L. Reginato, H. S. Hopkins, A. Debeling, W. Fritz, J. Meredith

This paper was prepared for submittal to the 12th International Symposium on Heavy Ion Fusion

Heidelberg, Germany

September 24-27, 1997

January 16, 1998

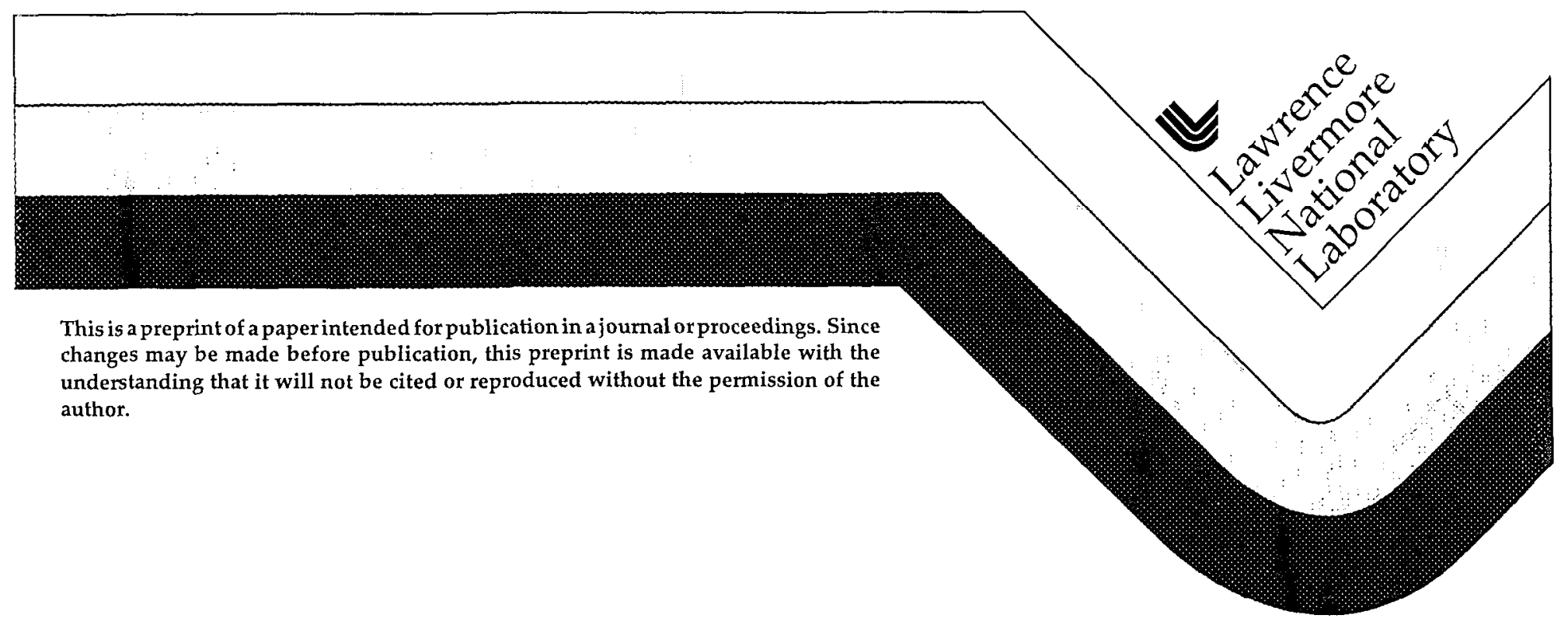




\section{DISCLAIMER}

This document was prepared as an account of work sponsored by an agency of the United States Government. Neither the United States Government nor the University of California nor any of their employees, makes any warranty, express or implied, or assumes any legal liability or responsibility for the accuracy, completeness, or usefulness of any information, apparatus, product, or process

disclosed, or represents that its use would not infringe privately owned rights. Reference herein to any specific commercial product, process, or service by trade name, trademark, manufacturer, or otherwise, does not necessarily constitute or imply its endorsement, recommendation, or favoring by the United States Government or the University of California. The views and opinions of authors expressed herein do not necessarily state or reflect those of the United States Government or the University of California, and shall not be used for advertising or product endorsement purposes. 


\title{
Status of Experiments Leading to a Small Recirculator
}

\author{
T. C. Sangster, J. J. Barnard, T. V. Cianciolo, G. D. Craig, A. Friedman, \\ D. P. Grote, E. Halaxa, R. L. Hanks, G. Kamin, H. C. Kirbie, \\ B. G. Logan, S. M. Lund, G. Mant, A. W. Molvik and W. M. Sharp
}

\section{Lawrence Livermore National Laboratory}

S. Eylon, D. Berners ${ }^{1}$, T. J. Fessenden, D. L. Judd and L. Reginato

Lawrence Berkeley National Laboratory

\author{
H. S. Hopkins \\ U. C. Berkeley
}

\begin{abstract}
A. Debeling, W. Fritz and J. Meredith
Bechtel Nevada Corporation
\end{abstract}

\begin{abstract}
A heavy ion linear induction accelerator is considered to be the leading driver candidate for an Inertial Fusion Energy reactor. To deliver a space-charge-dominated beam at the appropriate energy (several $\mathrm{GeV}$ ), such an accelerator would be several kilometers in length. Since total length has a strong influence on accelerator cost, we are considering the potential advantages and practical implementation of a recirculating induction accelerator. To address the critical scientific and technical challenges of a recirculating space-chargedominated heavy ion beam, we have begun to develop the elements of a scaled "small recirculator". An operating recirculator must demonstrate full beam control including multi-lap operation, beam insertion/extraction, acceleration and pulse compression. At present, experiments have been conducted using a $2 \mathrm{~mA}, 80 \mathrm{keV} \mathrm{K}^{+}$beam transported through a $45^{\circ}$ bend; experiments on a $90^{\circ}$ bend with five induction modulators will begin soon. This paper briefly summarizes the recirculator specifications and operational features and reports the latest experimental data as well as the developmental status of beam diagnostics.

PACS: 41.75.Ak, 41.75.Lx, 52.58.Hm

Keywords: accelerator, beams, diagnostics, recirculation, fusion, space-charge

${ }^{1}$ Present address: Stanford University

Primary Author: T. Craig Sangster

Lawrence Livermore National Laboratory

P.O. Box 808, L-481

Livermore, CA 94550

510-422-8176 (voice), 510-423-6319 (fax)

Sangster1@LLNL.Gov
\end{abstract}




\section{Introduction}

A number of accelerator-based inertial fusion energy (IFE) driver concepts are being evaluated on the basis of target specifications, complexity (technological risk) and cost [1]. All of these driver concepts share certain critical subsystems (e.g., high current sources, bunch compression and final focus) and all but one are variations on a conventional induction linac. The recirculator concept, based on a circular ion induction accelerator [2], introduces a number of new technical challenges while offering the prospect of significant cost reduction [3]. This paper reports on the status of an experimental program at Lawrence Livermore National Laboratory to assess the technical feasibility of the recirculation concept using a scaled "Small Recirculator". The most recent measurements show acceptable emittance growth and 100\% current transport of a $2 \mathrm{~mA}, 80 \mathrm{keV} \mathrm{K}^{+}$beam around a $45^{\circ}$ bend.

The functional requirements of the Small Recirculator include insertion, steering, acceleration, pulse compression and extraction - virtually the same functional requirements of a full-scale driver. Indeed, many of the dimensionless beam parameters were chosen to match those of an Amperc-class driver machinc. The development of the Small Recirculator is proceeding in parallel with a series of experiments designed to assess the feasibility and technical risk of the recirculation concept. When complete, the Small Recirculator will be the first circular ion induction accelerator and will provide a long path length platform (greater than $200 \mathrm{~m}$ for 15-lap operation) to address not only a host of beam dynamics issues (e.g., steering algorithms, longitudinal compression and confinement studies) but also to provide crucial benchmark data for sophisticated computational tools such as WARP3D [4] which will be used to design the first driverclass machine.

\section{Experimental Layout}

Figure 1 shows the $45^{\circ}$ layout as it existed during the summer of 1997. An $80 \mathrm{keV}$ pulser drives a Pierce electrode with a 1 " diameter potassium impregnated alumino-silicate source which is heated to approximately $900^{\circ} \mathrm{C}$. The source pulser, developed at LLNL [5], meets strict specifications for $\tau_{\text {rise }}$ and $\tau_{\text {fall }}$ (nominally $325 \mathrm{~ns}$ ) as well as repetition rate 
$(0.5 \mathrm{~Hz})$, flat-top ripple $(0.1 \%)$, droop $(<0.5 \%$ after $5 \mu \mathrm{s})$ and shot-to-shot variation in the flat-top voltage $(<1 \%)$. A space-charged-dominated beam of $\mathrm{K}^{+}$ions passes through a $1 \mathrm{~cm}$ diameter aperture (reducing the current to $2 \mathrm{~mA}$ ) into a matching section (see [6] for a more detailed description of the matching section) which contains a seven element electrostatic quadrupole strong focusing lattice, two steering "quadrupoles" (used as orthogonal dipoles) and various diagnostic ports. The matched beam is then delivered into a linear transport line with six permanent magnet quadrupoles and three diagnostic stations. The $45^{\circ}$ bend section is composed of five half lattice periods (HLPs), the fundamental building block of the Small Recirculator. Finally, a multi-purpose diagnostic tank is mounted at the end of the bend section approximately $7 \mathrm{~m}$ from the source.

Each HLP contains an electrostatic dipole which provides a nominal $9^{\circ}$ bend, a permanent magnet quadrupole (identical to the ones on the linear transport line), a diagnostic port, a capacitive probe (C-probe) and an ion pump (typical operational vacuum is a few $10^{-8}$ torr). In the near future (and on the full recirculator), every other IILP will also contain a sct of induction cores with a variable format modulator to provide longitudinal control, acceleration and pulse compression. Both the modulator and the pulser used to drive the electrostatic dipoles are described in the following section.

The diagnostics in the matching section include Faraday cups at both the entrance aperture and exit as well as two pair of vertical slit scanning devices which are used to measure the emittance and can be configured to measure a 2-D intensity profile. An optical pyrometer can also be mounted in the matching section to examine the temperature distribution across the face of the alumino-silicate source. The diagnostic stations in the linear transport line contain a pair of vertical slit scanners, a rotating wire scanner (RWS) [6] and an access port for the Gated Beam Imager (GBI) [6]. The HLP Cprobes provide beam position information $(\langle\mathrm{X}(\mathrm{t})\rangle,\langle\mathrm{Y}(\mathrm{t})\rangle)$ and will be discussed in the Measurements section. The diagnostic tank at the end of the $45^{\circ}$ bend contains a pair of vertical slit scanners, a Faraday cup and another access port for the GBI. This suite of diagnostics provides a rich set of beam characterization data and should be adequate to 
demonstrate the feasibility of recirculation in experiments planned for early 1998 on a fully instrumented $90^{\circ}$ bend.

\section{Longitudinal and Transverse Control}

The key to recirculating a space-charge-dominated ion beam is longitudinal and transverse beam control. The primary components of the beam control system are the induction modulators, the ramped electrostatic dipole pulsers and the beam position monitors (C-probes). The modulators have been designed to generate a 15-pulse burst at a frequency up to $91 \mathrm{kHz}$. The main pulse amplitude is nominally $500 \mathrm{~V}$ (the baseline acceleration voltage through each modulator) and is programmable for width, period and risetime. In addition, a trailing edge triangular "ear" pulse is added to the main square pulse in each modulator. The ear pulse varies in amplitude from 50 to $500 \mathrm{~V}$ with a variable risetime between $100 \mathrm{~ns}$ and $1 \mu \mathrm{s}$. The ear pulse is used to confine the head and tail of the beam which are being accelerated and decelerated, respectively, by space charge. The triangular ear pulses cause the tail of the beam to have a larger velocity than the head. With an ear pulse of the appropriate magnitude, the beam length can either be held constant (balancing space charge) or shortened (bunch compression).

The bending fields of the 40 electrostatic dipoles must increase in precise coordination with the increase in ion energy during acceleration. The dipoles are bipolar and the programmable dipole pulsers must deliver a voltage of approximately $\pm 6 \mathrm{kV}$ at the start of the pulse and smoothly ramp the voltage to approximately $\pm 28 \mathrm{kV}$ in $240 \mu \mathrm{s}$ (15-lap pulse residence time with acceleration from 80 to $320 \mathrm{keV}$ ). The variation between the delivered voltage and the required voltage should be around $1 \%$ to minimize the need for additional steering components. The dipole pulser circuit, developed at LBNL [7], is based on the amplification of a reference waveform using pulse width modulation in the feedback loop. The reference waveform is compared to the output voltage delivered to the dipole plates and the error signal adjusts a pulse width modulator to ensure that the high voltage output is a precise reproduction of the input reference. The feedback loop gain and response are adjusted at the amplifier stage. A single high voltage prototype has been tested with a capacitive load equivalent to eight Recirculator 
dipoles. The output voltage tracks the reference to better than $1 \%$ over the required range.

\section{Measurements}

The most straightforward beam characterization measurements are the emittance growth and the current transported as a function of distance from the sourcc. Figure 2 shows the beam current as a function of time measured in a Faraday cup at the end of the $45^{\circ}$ bend. The slight current droop is consistent with longitudinal expansion and the integrated current is, within the measurement error, identical to the current at the source. Figure 3 shows the vertical emittance $\left(\varepsilon_{y}\right)$ as a function distance from the source measured using slit scanning devices at the various diagnostic locations as well as the GBI at $45^{\circ}$. The emittance grows rapidly in the matching section then slows considerably in the straight section before rising again through the bend. A description of the expected emittance growth using a model of the Small Recirculator in WARP3D is given by Lund in [4]. The data in figure 3 are consistent with the theoretical predictions and the emittance budget for a 15-lap pulse through the Recirculator.

The GBI simultaneously measures both the in-plane $\left(\varepsilon_{\mathrm{x}}\right)$ and out-of-plane $\left(\varepsilon_{\mathrm{y}}\right)$ emittance; both are shown in figure 3 . This instrument has been specifically designed to operate with high current, low energy heavy ion beams. The emittance is measured in two orthogonal dimensions by creating a large number of emittance-dominated (rather than space-charge dominated) beamlets using a hole plate with $100 \mu \mathrm{m}$ diameter holes on $500 \mu \mathrm{m}$ centers. The beamlets drift approximately $16 \mathrm{~cm}$ before striking a metal cathode on the front of a micro-channel plate (MCP) coupled directly to a phosphor. The beamlet images on the phosphor are captured using a CCD camera. Based on the size and location of the beamlet images, all of the beam moments required to calculate the RMS emittance can be generated from a single beam pulse. Indeed, adequate images can be acquired for MCP gate times as short as $100 \mathrm{~ns}$ providing a detailed examination of the emittance as a function of time.

Although the design specifications and alignment tolerances of the Small Recirculator should be adequate for single-lap operation without steering, multi-lap 
operation will require a non-intercepting, nearly continuous monitor of the beam position with a resolution of approximately $100 \mu \mathrm{m}$. In reference [6], a 4-pad (upper left-right, lower left-right) capacitive probe located inside the permanent magnet quadrupoles on each HLP was proposed as the primary position diagnostic and preliminary prototype measurements suggested that the beam signal-to-noise ratio was adequate for the required resolution. Subsequent bench measurements using a precisely located wire with a current pulse inducing signals identical to those produced by the beam showed that the absolute position resolution of the $\mathrm{C}$-probe is less than $100 \mu \mathrm{m}$. Figure 4 shows the difference between the measured and the calculated wire location using the test bench setup. The resulting distribution is Gaussian with a width of approximately $70 \mu \mathrm{m}$. Additional operational tests with a prototype modulator were performed to assess the level of modulator signal pickup on the $\mathrm{C}$-probes. These tests indicated that two modifications (adding an aperture and a guard ring) to the C-probe design would be required to achieve the desired position resolution during acceleration by reducing the modulator commonmode pickup to approximately $2 \mathrm{mV}$ (approximately $1 \%$ of the beam signal).

\section{Conclusions}

A space-charge-dominated $\mathrm{K}^{+}$beam has been transported around a $45^{\circ}$ bend. The observed emittance growth is comparable to theoretical expectations and within the emittance budget allocated for 15-lap operation of the Small Recirculator. Functional tests of critical recirculator subsystems (high repetition rate induction modulators and electrostatic dipole pulsers, non-intercepting position monitors and control/steering algorithms) are currently underway on a $90^{\circ}$ configuration. These tests, as well as a series

of steering/beam control experiments, have been designed to conclusively demonstrate the feasibility of recirculation in the Small Recirculator and are planned for 1998.

\section{Acknowledgments}

This work was performed by LLNL and LBNL under the auspices of the US Department of Energy under contracts W-7405-ENG-48 and DE-AC03-76SF00098.

\section{References}


[1] J. J. Barnard etal., "Induction Accelerator Architectures for Heavy-Ion Fusion", proceedings of the 12th International Symposium on Heavy Ion Inertial Fusion, Heidelberg, Germany, Sept. 24-27, 1997.

[2] J. J. Barnard etal., UCRL-LR-108095, 1991.

[3] W. R. Meier, R. O. Bangerter and A. Faltens, "An Integrated Systems Model for Heavy Ion Drivers", proceedings of the 12th International Symposium on Heavy Ion Inertial Fusion, Heidelberg, Germany, Sept. 24-27, 1997.

[4] D. P. Grote, A. Friedman, I. Haber and S. Yu, "Three-Dimensional Simulations of High Current Beams in Induction Accelerators with WARP3d," Fus. Eng. Des. 32, 193 (1996); S. Lund etal., "Numerical Simulations of Intense-Beam Experiments at LLNL and LBNL", proceedings of the 12th International Symposium on Heavy Ion Inertial Fusion, Heidelberg, Germany, Sept. 24-27, 1997.

[5] M. J. Wilson, D. Goerz, R. Speer and R. Moal, "Heavy Ion Fusion Impulse Injector Design, Construction and Checkout", in preparation.

[6] T. J. Fessenden etal., Fus. Eng. and Des. 32, 267 (1996).

[7] D. P Berners and L. L. Reginato, "Arbitrary Waveform Generator for Electrostatic Dipoles in a Heavy Ion Recirculator", proceedings of the 1997 Particle Accelerator Conference, Vancouver, B.C., Canada, May 12-16, 1997.

\section{Figure Captions}

Figure 1. The layout of the $45^{\circ}$ bend experiment showing the relative locations of the various beam line components and diagnostics.

Figure 2. The ion current measured using a Faraday cup in the diagnostic tank following the $45^{\circ}$ bend for a $4 \mu$ s long beam pulse. The measurement indicates $100 \%$ current transport around the bend while the slight droop is consistent with longitudinal expansion due to space-charge.

Figure 3. The out-of-bend-plane emittance measured as a function of distance from the source. The open circles are measurements using pairs of slit-scanning devices; the solid boxes are similar measurements using a new alumino-silicate source. The solid triangles 
are the emittances calculated using the data from the GBI after the $45^{\circ}$ bend. The inbend-plane emittance is larger, as expected, while the out-of-bend-plane emittance is consistent with the slit measurement.

Figure 4. The absolute C-probe position resolution measured using a precisely located thin wire to induce signals similar to those produced by the beam. The upper panel (a) shows the correlation between the measured and calculated position over a range of \pm $2 \mathrm{~mm}$. The distribution of the differences between the measured and calculated positions is shown in the lower panel (b). The width of this distribution is approximately $70 \mu \mathrm{m}$, well within the required specifications for the $\mathrm{C}$-probe position resolution. 


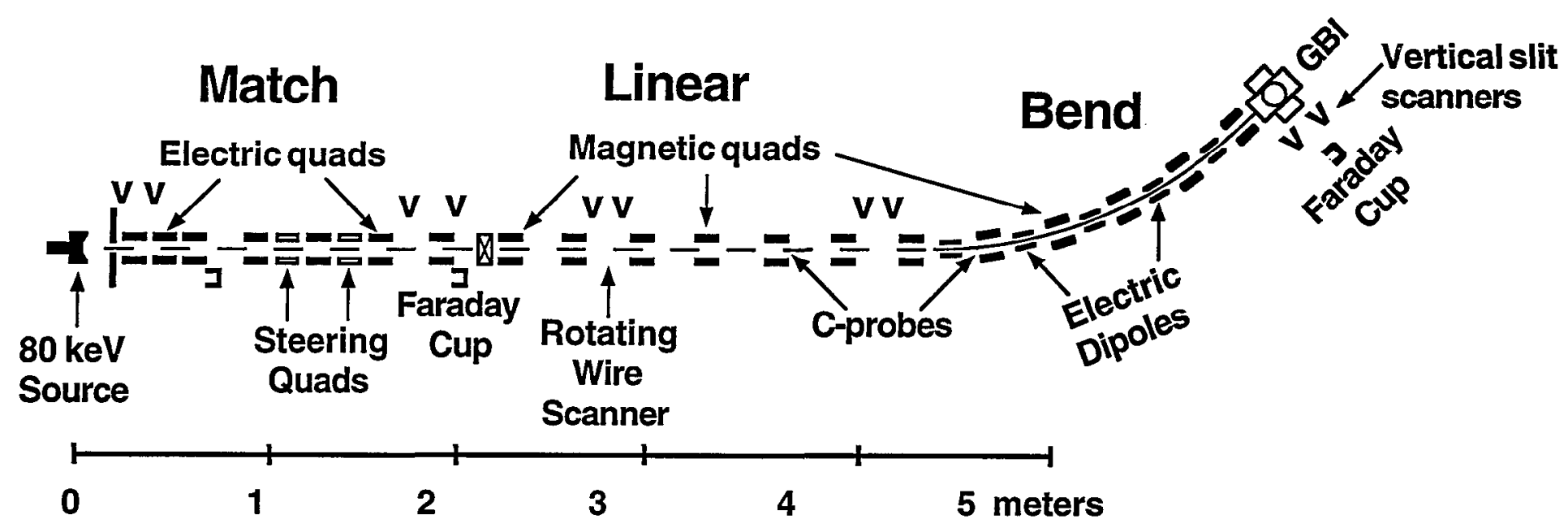

Figure 1 


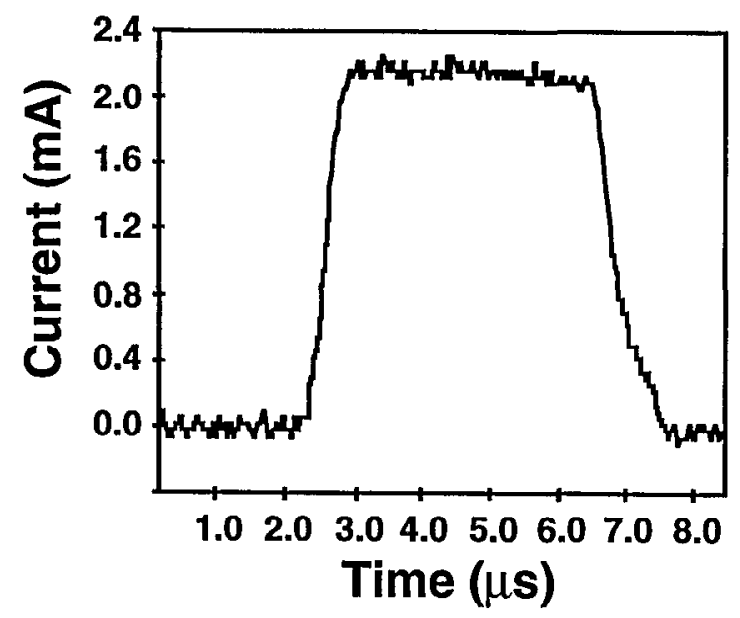

Figure 2 


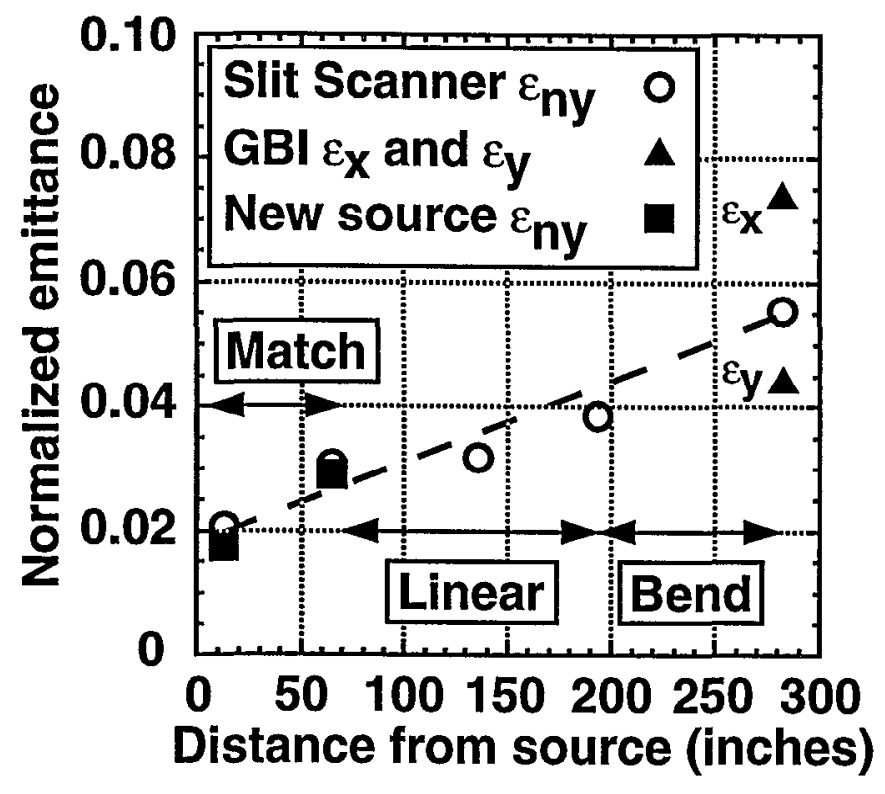

Figure 3 


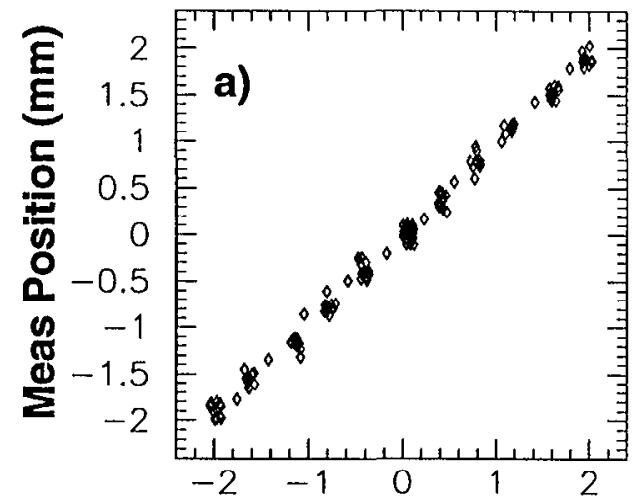

Calc Position ( $\mathrm{mm}$ )

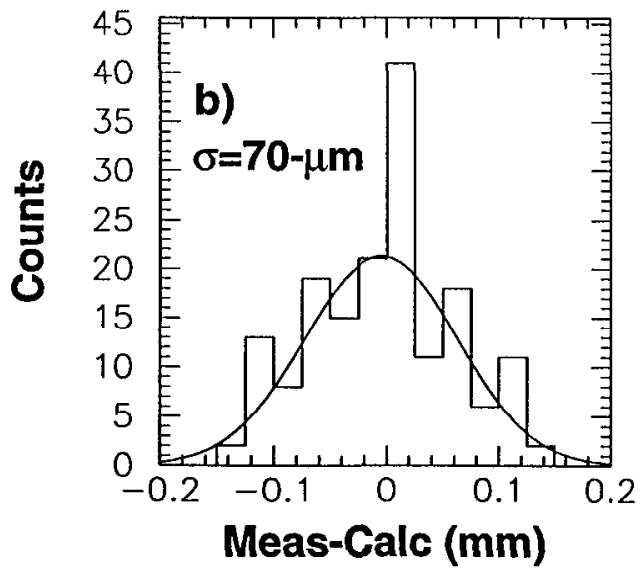

Figure 4 


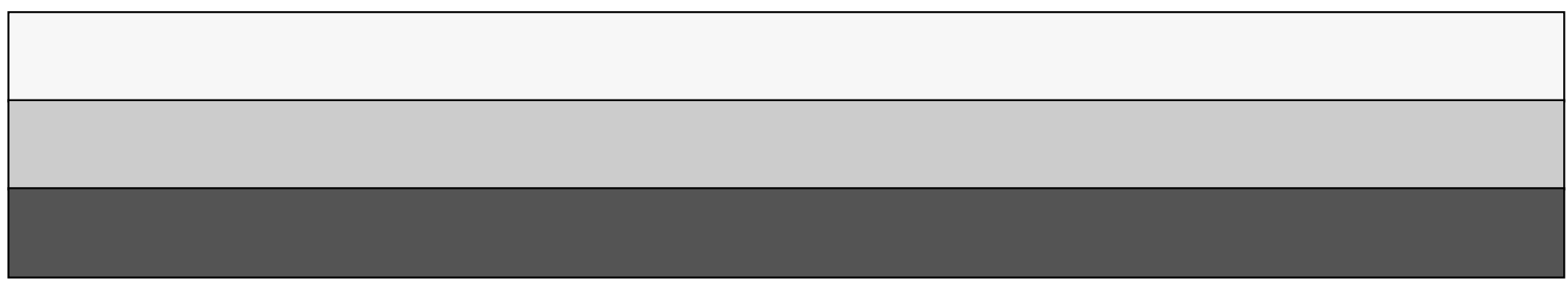

